\title{
Awareness of oral health in adults with congenital heart disease
}

\author{
Stefan Hollatz ${ }^{1 \#}$, Annette Wacker-Gussmann ${ }^{1 \#}$, Saskia Wilberg ${ }^{2}$, Matthias Folwaczny $^{2}$, Rhoia Neidenbach ${ }^{3}$, \\ Harald Kaemmerer ${ }^{3}$, Peter Ewert ${ }^{3}$, Renate Oberhoffer ${ }^{1}$
}

${ }^{1}$ Institute of Preventive Pediatrics, Faculty of Sport and Health Sciences, Technical University of Munich, München, Germany; ${ }^{2}$ Department of Conservative Dentistry and Periodontology, University Hospital, Ludwig-Maximilians University Munich, München, Germany; ${ }^{3}$ German Heart Center, Department of Pediatric Cardiology and Congenital Heart Disease, Technical University of Munich, München, Germany

Contributions: (I) Conception and design: S Hollatz, M Folwaczny, H Kaemmerer, R Oberhoffer; (II) Administrative support: S Hollatz; (III) Provision of study materials or patients: H Kaemmerer; (IV) Collection and assembly of data: S Wilberg, S Hollatz; (V) Data analysis and interpretation: S Hollatz; (VI) Manuscript writing: All authors; (VII) Final approval of manuscript: All authors.

\#These authors contributed equally to this work.

Correspondence to: Stefan Hollatz. Lehrstuhl für Präventive Pädiatrie, Georg- Brauchle Ring 60/62, D-80992 München, Germany.

Email: stef.hollatz1@gmail.com.

Background: Poor oral health is known to be a risk factor for infective endocarditis (IE) and acquired cardiovascular disease. Many patients with congenital heart disease (CHD) are at risk for developing IE. Excellent oral health is deemed essential to prevent IE, a potentially life-threatening condition, and therefore is important in adults with congenital heart defects (ACHD). As data on oral heath in ACHD are scarce, the current study aims to assess the prevalence of caries among ACHD and the knowledge of patients about oral health as a risk factor for IE and cardiovascular disease.

Methods: A cross-sectional study included ACHD who subsequently presented at the German Heart Center Munich. The participants completed a questionnaire assessing oral health and risk awareness. Additionally, as an indicator of caries prevalence, a dentist documented the DMFT index (sum of decayed, missing and filled teeth).

Results: The median age of the 112 participants was 31.5 (range, 18-77) years. Forty-three patients (38\%) were unaware of a correlation between oral health and heart disease. Twelve participants (11\%) did not know about poor oral health being a risk factor for cardiovascular diseases. Less than half of the surveyed patients knew the term endocarditis. An additional twelve patients (11\%) reported caveats against dental treatment because of their CHD. In twenty-three patients (21\%), caries was found at the dentine level. The mean DMFT index, representing caries prevalence, was $7.91( \pm 6.54)$. There was no correlation between the Bethesda criteria of CHD and the DMFT index $(\mathrm{P}=0.69)$. The DMFT index was higher in patients at high risk for IE $(\mathrm{P}<0.01)$. However, this difference in the caries score was mainly due to more extracted and filled teeth in the high-risk patients for IE, whereas the number of decayed teeth did not differ significantly $(\mathrm{P}=0.09)$.

Conclusions: The awareness of the importance of optimized oral health in ACHD needs to be improved.

Keywords: Adults with congenital heart defects (ACHD); congenital heart disease (CHD); endocarditis; oral health; prevention

Submitted Oct 26, 2018. Accepted for publication Dec 12, 2018.

doi: $10.21037 / \mathrm{cdt} .2019 .01 .01$

View this article at: http://dx.doi.org/10.21037/cdt.2019.01.01

\section{Introduction}

The risk of infective endocarditis (IE) increases considerably in patients with congenital heart defects (CHD) when compared to the general population. Remaining a lifethreatening condition with an in-hospital mortality of $16 \%$, the incidence of IE in adults with congenital heart defects (ACHD) is about 1.1 in 1,000 patient-years, but varies 
between the different types of CHD (1-3). Shear forces cause microlesions in the valvular or mural endocardium and endothelium, which lead to a reaction of the underlying matrix proteins with thromboplastin, fibrin and platelets (4). The resulting non-bacterial thrombotic endocarditis can become superinfected by bacteria which enter the blood stream through lesions in the mucosa of the oral cavity, the gastrointestinal or urogenital tract or the skin (5). Invasive medical procedures, including manipulations of infected tissue, may bear the risk of bacteremia as well as dental procedures with perforation of the oral mucosa and manipulation of the gingiva and periapical region of the teeth (6).

Bacteremia may also be caused by everyday activities such as chewing or brushing of the teeth $(3,7)$. The incidence of bacteremia with bacteria relevant to the pathogenesis of IE after brushing teeth is up to $23 \%$. Poor oral health in general is known to be an important risk factor for IE $(8,9)$.

Previously, antibiotic endocarditis prophylaxis (AEP) was recommended for patients at risk for IE prior to some invasive procedures. Due to the lack of sufficient evidence for its efficacy, the AEP guidelines were revised by the American Heart Association (AHA) and the European Society of Cardiology (ESC) in 2007. Since then, AEP is only recommended for patients with the highest risk $(5,6,10)$.

As there is no sufficient evidence for the efficacy of AEP as secondary prevention, primary prophylaxis of IE that includes the promotion of excellent oral health is becoming increasingly important. Various studies show an increased prevalence of caries in patients with CHD, which implies a risk for systemic effects (11-16). Other studies could not show a difference in oral health when patients with CHD and the heart-healthy population were compared $(17,18)$. However, none of these studies reflect the current status of oral health in ACHD.

IE guidelines outline the paramount importance of excellent oral health for the prevention of IE in CHD. However, patients know little about symptoms, risk factors and the individual risk of IE (19-25).

Particularly in ACHD, data on patients' awareness of the interrelation between poor oral health and cardiac diseases, dental prevention behavior and the prevalence of caries are limited and therefore aim of this study.

\section{Methods}

Patients with CHD were consecutively recruited from the out- and in-patient department of the German Heart Center Munich. They were asked to voluntarily participate in this cross-sectional, observational study. The study was conducted from July to August 2017. The inclusion criterion was a confirmed diagnosis of CHD in patients over 18 years old. The exclusion criterion was a lack of consent to the dental examination and to study participation.

The study was approved by the Medical School Ethics Committee of the Technical University Munich (study number 133/16S). All patients were informed in detail about the study and gave their informed consent.

The study participants were asked to complete a standardized questionnaire for oral health behavior and risk awareness. The questionnaire was designed for a previous study among children with CHD and adapted for adult patients (26). Patients with mental disability were only included in the study if a caretaker accompanied the patient and assisted in answering the questionnaire.

Additionally, a single dentist performed a standardized visual dental examination and documented the number of untreated decayed teeth and filled or missing teeth due to caries. To reflect caries prevalence in oral epidemiology, the decayed, missing and filled teeth (DMFT) index is a frequently used score (27). It is calculated as the sum of all DMFT in a patient. As the index also adds sufficiently treated teeth, it reflects the caries burden but not the actual caries activity in the study population.

The visual inspection of previously dried and cleaned teeth using the dentist's mirror is the key component to diagnose caries. However, in the approximal region of the teeth or under intact fissures, caries may not always be visually detectable and only diagnosed by $\mathrm{X}$-ray imaging. For study purposes only, the exposure to radiation was not ethically justifiable and dental X-ray examination was not performed. In the study setting, caries was only diagnosed when it reached the dentine level.

Patients' records were reviewed and medical diagnoses, surgical and medical treatments obtained. The CHD were classified according to the Bethesda criteria (28). Furthermore, it was recorded if the patient was at high risk for IE and had an indication for an AEP in accordance with current IE guidelines. Subgroup analyses aimed to identify special risk groups by detecting differences in the caries prevalence with respect to baseline characteristics such as gender, age, severity of the heart defect and the AEP indication. Moreover, variation of caries prevalence with reference to oral health behavior and risk awareness were assessed in further subgroup analyses. 
Table 1 Baseline characteristics in the study group

\begin{tabular}{|c|c|}
\hline Characteristics & Numbers \\
\hline Gender: female & $56(50 \%)$ \\
\hline Age, median and range, years & $31.5[18-77]$ \\
\hline $\mathrm{BMI}^{\dagger}$, mean $\pm \mathrm{SD}, \mathrm{kg} / \mathrm{m}^{2}$ & $24.3 \pm 4.8$ \\
\hline Out-patients & 95 (85\%) \\
\hline Inpatients & $17(15 \%)$ \\
\hline $\mathrm{CHD}$ of great complexity ${ }^{\ddagger}$ & $52(46 \%)$ \\
\hline CHD of moderate complexity ${ }^{\ddagger}$ & $44(39 \%)$ \\
\hline $\mathrm{CHD}_{\text {of simple complexity }}{ }^{\ddagger}$ & $16(14 \%)$ \\
\hline Patients with an AEP indication ${ }^{\S}$ & $52(46 \%)$ \\
\hline Patients with implanted pacemaker & $12(11 \%)$ \\
\hline Patients with ICD & $7(6 \%)$ \\
\hline \multicolumn{2}{|l|}{ Selected long-term medication } \\
\hline None & $36(32 \%)$ \\
\hline Vitamin $\mathrm{K}$ antagonists & $18(16 \%)$ \\
\hline $\begin{array}{l}\text { Non-vitamin K antagonist oral } \\
\text { anticoagulants (NOAC) }\end{array}$ & $16(14 \%)$ \\
\hline ACE inhibitors & $14(13 \%)$ \\
\hline Angiotensin receptor blocker & $7(6 \%)$ \\
\hline ASA & $4(4 \%)$ \\
\hline$\beta$-blocker & $38(34 \%)$ \\
\hline Diuretics $(\geq 1)$ & $24(21 \%)$ \\
\hline Pregnant women & $7(6 \%)$ \\
\hline Syndrome-associated CHD & $11(10 \%)$ \\
\hline Trisomy 21 & $3(3 \%)$ \\
\hline Microdeletion syndrome 22q11 & $3(3 \%)$ \\
\hline Holt-Oram syndrome & $1(1 \%)$ \\
\hline Shone syndrome (incomplete) & $1(1 \%)$ \\
\hline Marfan syndrome & $1(1 \%)$ \\
\hline Noonan syndrome & $1(1 \%)$ \\
\hline Williams-Beuren syndrome & $1(1 \%)$ \\
\hline
\end{tabular}

${ }^{\dagger}$, pregnant women were excluded; ${ }^{\ddagger}$, Bethesda criteria of CHD;

\$, classified high-risk patients in accordance with the AHA guidelines on IE. CHD, congenital heart disease; AHA, American Heart Association; IE, infective endocarditis.

The caries prevalence in ACHD was also compared to the available data on the heart-healthy 35- to 44-year-old German population. The according age cohort was selected from the study population.

\section{Statistics}

All data were analyzed using SPSS $^{\circledR}$ (version 22.0, IBM $^{\circledR}$ Corporation, USA). Data were shown as absolute and relative frequencies, means and standard deviations (SD) were calculated and tests for significance performed (Chisquare tests, the $t$-test for normally distributed values, the Mann-Whitney U test or Kruskal-Wallis test as nonparametric tests, the Spearman correlation coefficient). Age as an important confounder of the DMFT index was taken into account by using a linear regression analysis when performing subgroup analyses. $\mathrm{P}$ values below 0.05 were considered statistically significant.

\section{Results}

Fifty-six women and 56 men, mainly with CHD of great $(46 \%)$ or moderate $(39 \%)$ complexity and aged 18 to 77 years were included in the study. Eleven patients (10\%) had syndrome-associated CHD.

There was no documented long-term medication use such as anticholinergic drugs, tricyclic antidepressants, cyclosporine or methotrexate which predisposes to bad oral health. Only one patient had diabetes mellitus, a disease known to come along with a higher prevalence of oro-dental comorbidities. The baseline characteristics are summarized in Table 1.

Fifty-two patients with CHD (46\%) met criteria for an AEP prior to certain dental treatments. These patients were considered at high-risk for IE. Table 2 shows the AEP indications in accordance with the AHA guidelines and the frequency in the study population (5). There were 7 documented cases of previous IE in the study population.

\section{Knowledge of oral bealth and questionnaire results}

The participants were asked if they were aware of a correlation between oral health and the development of acquired cardiac diseases, but they did not have to specify the cardiac diseases. In total, 43 patients $(38 \%)$ were unaware of any correlation between oral health and cardiac disease and an additional 39 (35\%) did not feel sufficiently well informed about the link.

Twelve of the surveyed ACHD (11\%) stated that they did not think that poor oral health was a risk factor for acquired cardiac diseases and another $22(20 \%)$ were not sure if 
Table 2 High-risk patients with an indication for an AEP in accordance with AHA guidelines (5) and absolute and relative frequencies in the study population

\begin{tabular}{lcc}
\hline Cardiac condition & Absolute frequency & Relative frequency (\%) \\
\hline Prosthetic cardiac valve or prosthetic material used for cardiac valve repair & 23 & 4 \\
Previous IE & 3 & 3.6 \\
Previous IE in patients with prosthetic cardiac valve or prosthetic material & 2 & 2.7 \\
Uncorrected cyanotic CHD & 9 & 1.8 \\
Palliative shunt or conduit & 7 & 8.0 \\
Residual defects of prosthetic material inhibiting its endothelialization & 4 & 3.3 \\
Less than 6 months after interventional or cardiothoracic procedure with & 3.6 \\
implantation of foreign material & 0 \\
Cardiac transplantation with cardiac valvulopathy & 0 \\
\hline
\end{tabular}

AEP, antibiotic endocarditis prophylaxis; CHD, congenital heart disease; AHA, American Heart Association; IE, infective endocarditis.

poor oral health was a risk factor for the development of cardiovascular disease.

Sixty patients (54\%) did not know the term endocarditis.

Forty-two patients (38\%) attached special importance to their dental hygiene because of their CHD, while 52 ACHD (46\%) did not especially care about their dental hygiene.

For caries prevention, the German Society for Conservative Dentistry recommends brushing teeth at least twice daily and regular flossing of the interdental spaces (29). In the study population, 93 (84\%) brushed their teeth twice or three times a day but $18(16 \%)$ brushed their teeth less than twice a day. Fifty-seven patients (53\%) never flossed their teeth and $17(16 \%)$ only once a month. For dental prophylaxis visits to the dentist are recommended at least once a year (30). Twelve patients (11\%) saw their dentist less than once a year or never. Thirty-eight patients (35\%) had never had a professional dental cleaning. Table 3 shows the questions and answers of all study participants and the high-risk patients.

\section{High-risk patients}

Among the patients at high risk for IE who met criteria for an AEP, 22 (42\%) felt that they were well informed about the link between oral and possible cardiac complications. While 15 (29\%) of the patients at high risk for IE were unaware of the link, another $15(29 \%)$ did not feel sufficiently well informed. Only 27 (52\%) of the high-risk patients attached special importance to their dental hygiene because of CHD. Even among the high-risk patients, 22 of them (42\%) did not know the term endocarditis. Compared to the general population patients at high risk for IE were more often aware of the interrelation between poor oral health and cardiac diseases, attached more importance to dental hygiene and knew the term endocarditis more frequently (Table 3).

Furthermore, 10 (19\%) of the patients with an indication for an AEP stated that their cardiologist did not inform them about the necessity of antibiotic administration prior to certain dental treatments. Of all patients with an AEP indication, $8(15 \%)$ did not possess a heart disease record card for the prevention of IE and another 11 (22\%) did not show this wallet card to their treating dentist.

Among the high-risk patients, 8 (15\%) worried about dental treatment causing cardiac problems, whereas only $4(8 \%)$ without an AEP indication reported this fear. Only 2 patients were afraid that daily dental hygiene could cause cardiac problems.

\section{DMFT index of all patients}

The mean DMFT index of the 112 study participants was $7.91( \pm 6.54)$ (Figure 1). Nine patients $(8 \%)$ were caries-free (DMFT index $=0)$. Twenty-three patients $(21 \%)$ had at least one untreated decayed tooth and, on average, $0.33 \pm 0.76$ teeth were found decayed [95\% confidence interval (CI): 0.2-0.5].

A representative DMFT index in the German population was only available for certain age groups (31). Therefore, we selected the 35- to 44-year-old German population as control group and compared the data with the results from the age-matched study participants. Twenty-three study 
Table 3 Excerpt from the questionnaire assessing oral health and risk awareness in ACHD and answers of all study participants and high-risk patients

\begin{tabular}{|c|c|c|c|c|}
\hline \multirow[b]{2}{*}{ Questions } & \multicolumn{2}{|c|}{ All ACHD (n=112) } & \multicolumn{2}{|c|}{ High-risk patients $(n=52)$} \\
\hline & $\begin{array}{l}\text { Absolute } \\
\text { frequency }\end{array}$ & $\begin{array}{c}\text { Relative } \\
\text { frequency (\%) }\end{array}$ & $\begin{array}{l}\text { Absolute } \\
\text { frequency }\end{array}$ & $\begin{array}{c}\text { Relative } \\
\text { frequency (\%) }\end{array}$ \\
\hline
\end{tabular}

1. Do you think that poor oral health is a risk factor for cardiac complications?
a. Yes
b. No
c. I do not know

78

12

22

2. Are you aware of a correlation between oral health and possible cardiac diseases?
a. Yes, I feel sufficiently well informed about the link
b. Yes, but I do not feel sufficiently well informed about the link
c. No

39

43

26.8

34.8

22

42.3

c. No

3. Is dental hygiene especially important for you because of your CHD?
a. Yes
b. No
c. I do not know

42

52

18

38.4

37

71.2

10.7

5

9.6

9.6

10

19.2

4. Did you have to postpone dental treatment or follow-up appointments for more than 6 months because of your CHD?
a. Yes
b. No
C. I do not know

$\begin{array}{cccc}5 & 4.5 & 5 & 9.6 \\ 102 & 91.1 & 43 & 82.7 \\ 5 & 4.5 & 4 & 7.7\end{array}$

5. Are you afraid of dental treatment because of your CHD?
a. Yes
b. No
c. I do not know

12

10.7

8

15.4

97

3

86.6

42

80.8

6. Are you worried about dental hygiene impairing your heart?
a. Yes
b. No
C. I do not know

2

2.7

2

3.8

104

1.8

2

3.8

6

92.9

48

92.3

7. Do you have a heart disease record card?
a. Yes, and my dentist is aware of it
b. Yes, but my dentist has not seen it
c. No

65

5.4

2

3.8

24

60.2

31

62.0

19

22.2

11

22.0

Table 3 (continued) 
Table 3 (continued)

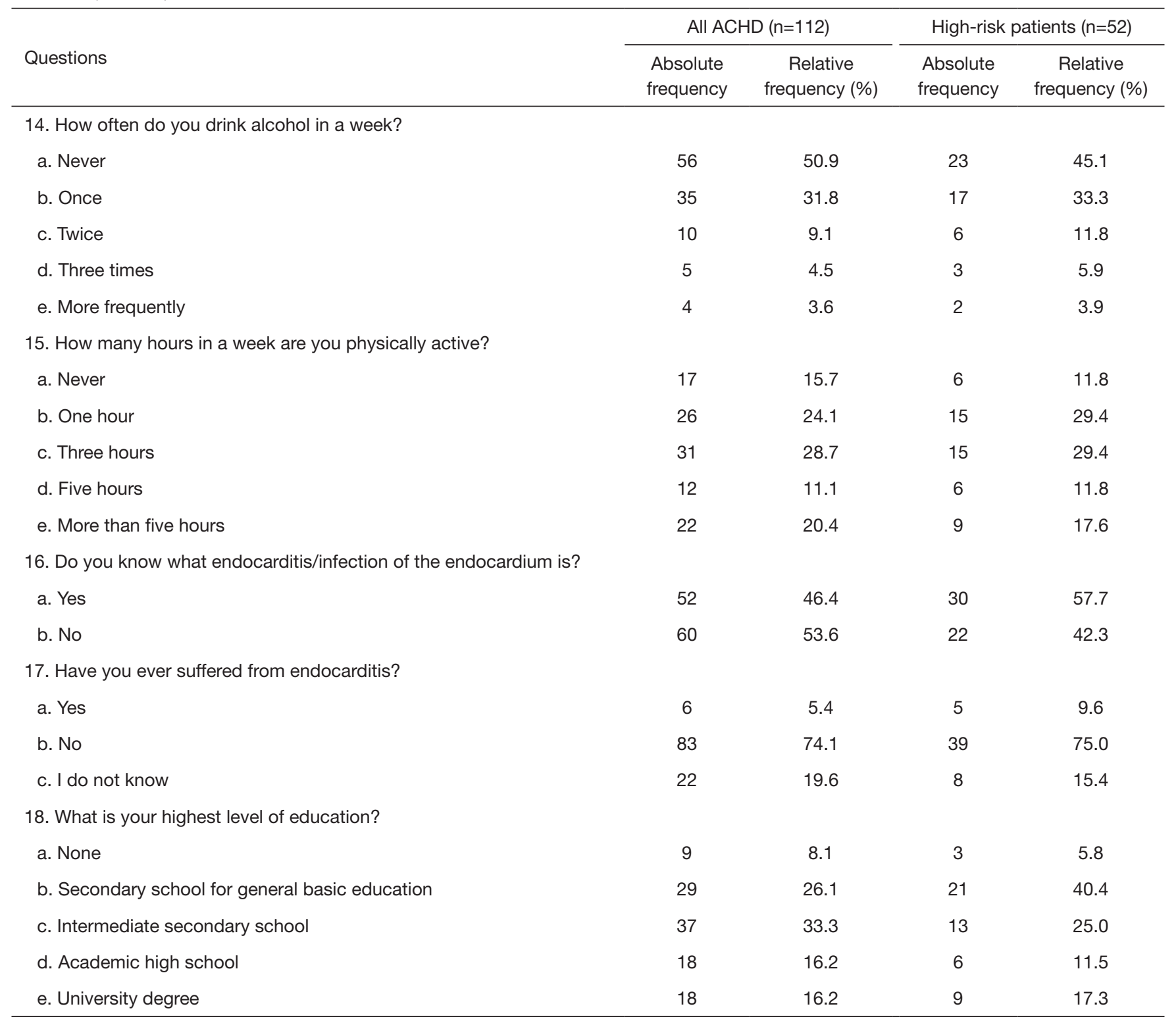

ACHD, adults with congenital heart defects.

participants fitted the age span. The mean DMFT index was $9.04 \pm 5.95$ (95\% CI: 6.5 to 11.6 ) in the study group and 11.2 in the control group. The difference was statistically not significant.

The number of untreated decayed teeth in the control group is described as 0.5 teeth on average. This number is very similar to the average 0.48 untreated decayed teeth found in 35- to 44-year-old ACHD. Also, the number of decayed teeth in all 112 ACHD did not differ significantly from the heart-healthy population.

\section{Association between the DMFT index and patient characteristics/ knowledge}

As the DMFT index also adds sufficiently treated teeth, its values never decrease during a lifetime. Therefore, age is an important confounder of the DMFT index and effects of age needed to be adjusted using linear regression analyses $\left(\mathrm{R}^{2}=0.389\right)$.

There was no significant gender difference in the DMFT index $(\mathrm{P}=0.152)$. 

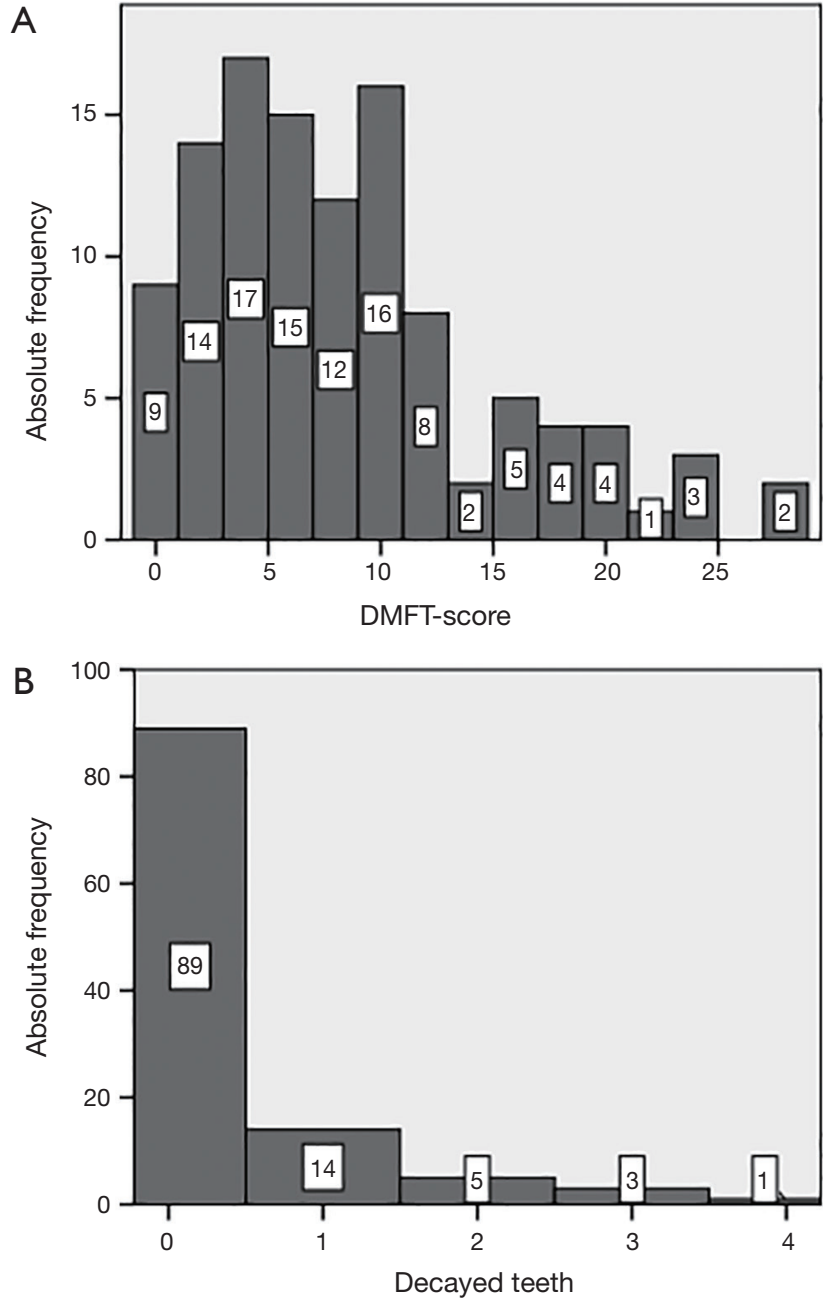

Figure 1 Distribution of the DMFT index (A) and number of untreated decayed teeth (B). DMFT, decayed, missing and filled teeth.

The DMFT index was significantly higher in patients who met criteria for an AEP than in patients without an AEP indication $(\mathrm{P}<0.01$, Figure 2$)$ but no significant difference in untreated decayed teeth $(\mathrm{P}=0.086)$. Significantly more teeth were extracted or filled due to caries $(\mathrm{P}<0.01)$.

There was no significant association between the DMFT index and the severity of the CHD after adjusting for age $(\mathrm{P}=0.694)$.

Syndromes associated with CHD were found in the records of 11 patients. The DMFT index of these patients did not differ significantly from that of patients without syndrome-associated CHD ( $\mathrm{P}=0.092)$. Furthermore, there was no significant correlation between the DMFT index and the level of education, awareness of the interrelation of

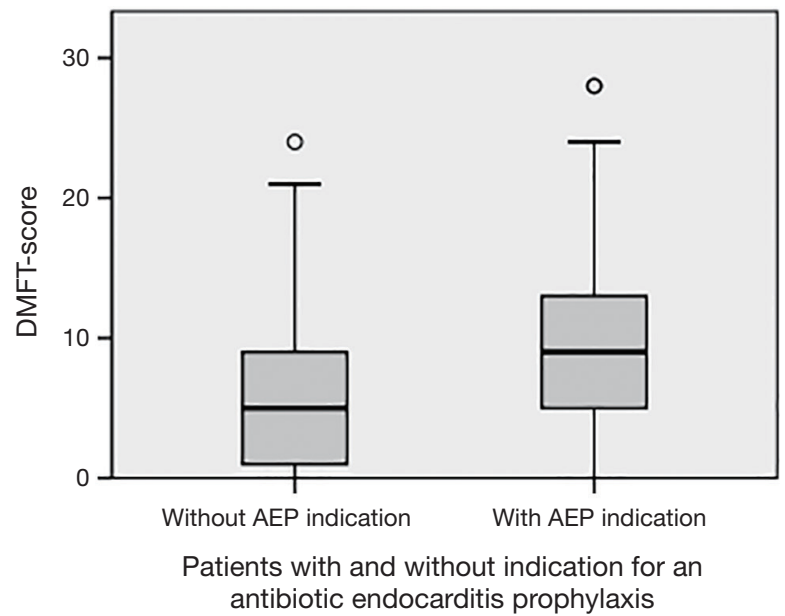

Figure 2 DMFT index of patients with and without an AEP indication. DMFT, decayed, missing and filled teeth; AEP, antibiotic endocarditis prophylaxis.

oral health and cardiac complication, knowledge of the term endocarditis, fears of dental treatment or special compliance with dental hygiene.

\section{Discussion}

The main finding of this study on ACHD is the lack of knowledge on the interrelation between poor oral health and cardiovascular diseases. Furthermore, there was no evidence for an increased caries prevalence in ACHD compared with the heart-healthy population. Nevertheless, one in five patients with CHD was found to have at least one untreated decayed tooth. This indicates that dental health must be optimized in precarious ACHD.

The number of missing and filled teeth due to caries and the DMFT index were higher in patients with an indication for an AEP. As these high-risk patients were more often aware of the importance of good oral health, this may have led to more frequent dental treatments. However, the number of untreated decayed teeth did not differ significantly. Therefore, it remains uncertain if the difference in the DMFT index was due to more frequent dental treatment or an increased caries prevalence in highrisk patients.

However, various studies describe scenarios which could lead to an increased prevalence of poor oral health in patients with CHD, implying a systemic hazardous effect (11-15). Developmental hypoplasia of the enamel, malocclusion and crowding are more prevalent in children 
with CHD and can lead to a higher incidence of caries $(14,21)$. Drugs can have negative side effects on oral health by causing xerostomia or vomiting, for example, and if administered as sugary syrups (18). Furthermore, patients with CHD consult physicians frequently and are disturbed by hospital stays. These facts may lead to an attitude of avoidance towards dentists. Moreover, patients may fear the negative effects of dental procedures on their heart. They might also be skeptical about taking antibiotics and delay or avoid dental visits. However, in this study only one in ten patients reported worrying about a possible cardiac impairment caused by a dental treatment.

Consensus in the current guidelines on IE is the paramount importance of excellent oral health aiming to prevent hematogenous spread of bacteria from the oral cavity and posing a risk to CHD patients $(5,6,32,33)$. Primary prophylaxis of poor oral health includes avoiding cariogenic food (sugar), brushing teeth at least twice a day and flossing teeth regularly (29). In the current study, more than half of the surveyed patients never flossed their teeth and one in six brushed his/her teeth less than twice a day. Additionally, adequate prophylaxis involves keeping dental appointments on a regular basis. However, one in ten surveyed patients sees the dentist less than once a year. This limited utilization of dental prophylactic measures should be remedied especially in patients at high risk for IE.

Our data show that the surveyed ACHD were not informed well enough about the importance of good oral health. Almost three in four $(73 \%)$ reported that their knowledge of a correlation between oral health and cardiac complications was either inadequate or non-existent. Even among patients with the highest risk for an IE, the risk awareness was insufficient. These findings among ACHD agree with prior studies on patient information on IE among children with CHD and their parents $(20-23,25,26)$. However, patient education and disease awareness are especially important in chronic diseases like CHD and they must be promoted by congenital cardiologists, general practitioners and dentists. In patients with CHD, better patient education on IE can lead to more frequent dental visits (34).

In this study, more than one in five patients who require an AEP prior to certain dental treatments did not report needing the antibiotic prophylaxis. This indicates a remarkable misinformation on the need of AEP in patients at high risk for IE. Patient education must be improved.

With the aim to communicate the necessity of an AEP adequately to other physicians or dentists, patients should have an endocarditis wallet card for the prevention of IE and show it to their treating dentist. There is a need to optimize its use, as more than one in three patients with an AEP indication either did not have such a wallet card or did not show it to their dentist.

\section{Limitations of the study}

In the study setting, scaling could not be performed prior to the examination due to the risk of causing a bacteremia in patients at risk for IE and initial carious lesions were not diagnosed. A possible underestimation of caries prevalence cannot be ruled out due to missing $\mathrm{X}$-ray examination and detection of hidden caries.

Furthermore, as oral health is marked with less shame in patients with generally good oral health, they may have more frequently agreed to voluntarily participate in the study than patients with poor oral health. Another selection bias could be that patients with dentist phobia may have poorer oral health. These patients may tend to refuse a voluntary dental examination, which may have led to an underrepresentation of the more severe cases of caries.

Besides caries, poor oral health is also caused by gingivitis or periodontitis, highly prevalent diseases that should also be considered in future investigation assessing oral health in ACHD.

The validity and reliability of the questionnaire were not tested.

It must also be considered that the study population presented in a tertiary center of medical attention and did not reflect the distribution of ACHD in the general population.

\section{Conclusions}

As the number of patients with CHD reaching adulthood is increasing due to improvements in medical and surgical care, a multidisciplinary preventive approach to potentially life-threatening conditions is necessary (35-38). It is essential to optimize disease awareness in order to encourage patients' self-management. All IE guidelines highlight the major importance of excellent oral health in high-risk patients. Therefore, the interdisciplinary team of general practitioners, adult and pediatric cardiologists and dentists must increase efforts to promote adequate and specific health education. Aiming to improve the knowledge of their disease, to raise risk awareness and reinforce good health-related behavior, structured patient information and individual recommendations are of clinical importance. In 
order to adequately face the potentially hazardous effects of poor oral health in patients at risk, all primary prevention options must be exploited. Furthermore, future research on oral health in ACHD should assess the prevalence of gingivitis and periodontitis as an important risk factor for bacteremia occurring in everyday life.

\section{Acknowledgments}

The work was supported by the Institute of Preventive Pediatrics, Faculty of Sport and Health Sciences, Technical University of Munich, Germany and the Department of Conservative Dentistry and Periodontology, University Hospital, Ludwig-Maximilians University Munich, Germany.

\section{Footnote}

Conflicts of Interest: The authors have no conflicts of interest to declare.

Ethical Statement: The study was approved by the Medical School Ethics Committee of the Technical University Munich (study number 133/16S). All procedures performed in studies involving human participants complied with the ethical standards of the institutional and national research committee and with the 1964 Declaration of Helsinki and its later amendments or comparable ethical standards, and informed consent was obtained from all study participants.

\section{References}

1. Tutarel O, Alonso-Gonzalez R, Montanaro C, et al. Infective endocarditis in adults with congenital heart disease remains a lethal disease. Heart 2018;104:161-5.

2. Verheugt CL, Uiterwaal CS, van der Velde ET, et al. Turning 18 with congenital heart disease: prediction of infective endocarditis based on a large population. Eur Heart J 2011;32:1926-34.

3. Moreillon P, Que YA. Infective endocarditis. Lancet 2004:363:139-49.

4. Knirsch W, Nadal D. Infective endocarditis in congenital heart disease. Eur J Pediatr 2011;170:1111-27.

5. Wilson W, Taubert KA, Gewitz M, et al. Prevention of infective endocarditis: guidelines from the American Heart Association: a guideline from the American Heart Association Rheumatic Fever, Endocarditis, and Kawasaki Disease Committee, Council on Cardiovascular Disease in the Young, and the Council on Clinical Cardiology, Council on Cardiovascular Surgery and Anesthesia, and the Quality of Care and Outcomes Research Interdisciplinary Working Group. Circulation 2007;116:1736-54.

6. Habib G, Lancellotti P, Antunes MJ, et al. 2015 ESC Guidelines for the management of infective endocarditis: The Task Force for the Management of Infective Endocarditis of the European Society of Cardiology (ESC). Endorsed by: European Association for Cardio-Thoracic Surgery (EACTS), the European Association of Nuclear Medicine (EANM). Eur Heart J 2015;36:3075-128.

7. Roberts GJ. Dentists are innocent! "Everyday" bacteremia is the real culprit: a review and assessment of the evidence that dental surgical procedures are a principal cause of bacterial endocarditis in children. Pediatr Cardiol 1999;20:317-25.

8. Forner L, Larsen T, Kilian M, et al. Incidence of bacteremia after chewing, tooth brushing and scaling in individuals with periodontal inflammation. J Clin Periodontol 2006;33:401-7.

9. Lockhart PB, Brennan MT, Sasser HC, et al. Bacteremia associated with toothbrushing and dental extraction. Circulation 2008;117:3118-25.

10. Naik RJ, Patel NR, Wang M, Shah NC. Infective endocarditis prophylaxis: current practice trend among paediatric cardiologists: are we following the 2007 guidelines? Cardiol Young 2016;26:1176-82.

11. Cantekin K, Yilmaz Y, Cantekin I, et al. Comprehensive dental evaluation of children with congenital or acquired heart disease. Cardiol Young 2013;23:705-10.

12. Garg SA TN, Baliga SM, Bhatiya PV. Estimation of salivary nitric oxide levels in children with congenital heart diseases. Indian J Dent 2015;6:65-8.

13. Pimentel EL, Azevedo VM, Castro Rde A, et al. Caries experience in young children with congenital heart disease in a developing country. Braz Oral Res 2013;27:103-8.

14. Hallett KB, Radford DJ, Seow WK. Oral health of children with congenital cardiac diseases: a controlled study. Pediatr Dent 1992;14:224-30.

15. Franco E, Saunders CP, Roberts GJ, et al. Dental disease, caries related microflora and salivary IgA of children with severe congenital cardiac disease: an epidemiological and oral microbial survey. Pediatr Dent 1996;18:228-35.

16. Sivertsen TB, Aßmus J, Greve G, et al. Oral health among children with congenital heart defects in Western Norway. Eur Arch Paediatr Dent 2016;17:397-406.

17. Balmer R, Booras G, Parsons J. The oral health of children considered very high risk for infective endocarditis. Int $\mathrm{J}$ 
Paediatr Dent 2010;20:173-8.

18. da Fonseca MA, Evans M, Teske D, et al. The impact of oral health on the quality of life of young patients with congenital cardiac disease. Cardiol Young 2009;19:252-6.

19. Knirsch W, Hassberg D, Beyer A, et al. Knowledge, compliance and practice of antibiotic endocarditis prophylaxis of patients with congenital heart disease. Pediatr Cardiol 2003;24:344-9.

20. da Silva DB, Souza IP, Cunha MC. Knowledge, attitudes and status of oral health in children at risk for infective endocarditis. Int J Paediatr Dent 2002;12:124-31.

21. Balmer R, Bu'Lock FA. The experiences with oral health and dental prevention of children with congenital heart disease. Cardiol Young 2003;13:439-43.

22. Knöchelmann A, Geyer S, Grosser U. Maternal understanding of infective endocarditis after hospitalization: assessing the knowledge of mothers of children with congenital heart disease and the practical implications. Pediatr Cardiol 2014;35:223-31.

23. Löbel A, Geyer S, Grosser U, et al. Knowledge of congenital heart disease of mothers: presentation of a standardized questionnaire and first results. Congenit Heart Dis 2012;7:31-40.

24. Moons P, De Volder E, Budts W, et al. What do adult patients with congenital heart disease know about their disease, treatment, and prevention of complications? A call for structured patient education. Heart 2001;86:74-80.

25. Saunders CP, Roberts GJ. Dental attitudes, knowledge, and health practices of parents of children with congenital heart disease. Arch Dis Child 1997;76:539-40.

26. Koerdt S, Hartz J, Hollatz S, et al. Dental prevention and disease awareness in children with congenital heart disease. Clin Oral Investig 2018;22:1487-93.

27. Larmas M. Has dental caries prevalence some connection with caries index values in adults? Caries Res 2010;44:81-4.

28. Warnes CA, Liberthson R, Danielson GK, et al. Task Force 1: the changing profile of congenital heart disease in adult life. J Am Coll Cardiol 2001;37:1170-5.

29. Deutsche Gesellschaft für Zahnerhaltung DGfZ-, Mundund Kieferheilkunde S2k-Leitlinie Kariesprophylaxe bei bleibenden Zähnen - grundlegende Empfehlungen AWMF-Registernummer: 083-021. 2016.

30. Micheelis W, Schiffner U. Vierte Deutsche Mundgesundheitsstudie (DMS IV). Institut der deutschen Zahnärzte, 2006.

31. Jordan RA, Micheelis W, Cholmakow-Bodechtel C, et al. Fünfte Deutsche Mundgesundheitsstudie (DMS V). 35 ed. Köln: Deutscher Zahnärzte Verlag DÄV, 2016.

32. Naber CK, Al-Nawas B, Baumgartner H, et al. Prophylaxe der infektiösen Endokarditis. Der Kardiologe 2007;1:243-50.

33. NICE-Short_Clinical_Guidelines_Technical_Team. Prophylaxis against infective endocarditis: antimicrobial prophylaxis against infective endocarditis in adults and children undergoing interventional procedures. National Institute for Health and Clinical Excellence, London, 2008.

34. Janssens A, Goossens E, Luyckx K, et al. Exploring the relationship between disease-related knowledge and health risk behaviours in young people with congenital heart disease. Eur J Cardiovasc Nurs 2016;15:231-40.

35. Neidenbach R, Kaemmerer H, Pieper L, et al. Erwachsene mit angeborenen Herzfehlern - Eklatante Versorgungslücke? Dtsch Med Wochenschr 2017;142:301-3.

36. Neidenbach R, Nagdyman N, Oberhoffer R, et al. Angeborene Herzfehler im Langzeitverlauf - Lebenslange Betreuung der Patienten. Pädiatrie 2017;29(6).

37. Neidenbach R,Schelling J, Pieper L, et al. Sind Erwachsene mit angeborenen Herzfehlern ausreichend versorgt? Zeitschrift für Herz thorax und Gefasschirurgie 2017;31:S228-40.

38. Kaemmerer H, Bauer U, de Haan F, et al. Recommendations for improving the quality of the interdisciplinary medical care of grown-ups with congenital heart disease (GUCH). Int J Cardiol 2011;150:59-64.
Cite this article as: Hollatz S, Wacker-Gussmann A, Wilberg S, Folwaczny M, Neidenbach R, Kaemmerer H, Ewert P, Oberhoffer R. Awareness of oral health in adults with congenital heart disease. Cardiovasc Diagn Ther 2019;9(Suppl 2):S281S291. doi: 10.21037/cdt.2019.01.01 